\title{
Parâmetros genéticos e tendências genéticas e fenotípicas para escores visuais na fase pós-desmama de bovinos da raça Aberdeen Angus
}

\author{
Genetic parameters and genetic and phenotypic trends for post weaning visual scores in Aberdeen \\ Angus breed cattle
}

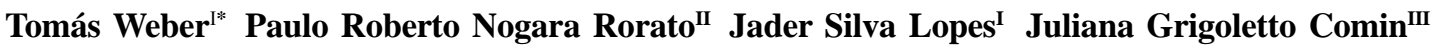 \\ Mariana de Almeida Dornelles ${ }^{\mathrm{III}}$ Ronyere Olegário de Araújo ${ }^{\mathrm{I}}$
}

\section{RESUMO}

Neste trabalho, foram avaliados, na fase de pósdesmama, 28.349 animais da raça Aberdeen Angus, nascidos entre os anos de 1993 e 2003 e criados em 141 fazendas. Os objetivos deste trabalho foram estimar parâmetros genéticos e avaliar a tendência genética e a fenotípica para os escores de avaliação visual (EVs), conformação $(C)$, precocidade $(P)$, musculatura (M) e tamanho (T). Os componentes de (co)variância foram estimados por REML, utilizando um modelo animal. As estimativas de herdabilidade foram: 0,13; 0,11 ; 0,16 e 0,13 para $C, P, M$ e T, respectivamente. As correlações genéticas obtidas entre os escores visuais variaram de 0,01 a 0,92. As tendências genéticas e fenotípicas para $C, P$, $M$ e T (pontos/ano) foram: 0,0054 e 0,0189; 0,0035 e-0,0013; 0,0057 e 0,0217 e 0,0026 e-0,0016, respectivamente. As herdabilidades estimadas sugerem baixa resposta à seleção direta. As correlações genéticas entre os EVs foram altas entre $C, P$ e $M(0,79$ a 0,92) e foram baixas entre estes e $T(0,01$ a $0,30)$. As tendências genéticas mostram que a seleção está promovendo ganho genético de pequena magnitude, porém, as tendências fenotípicas, com valores negativos para algumas características, indicam que deve ser dada mais atenção para as condições ambientais.

Palavras-chave: avaliação genética, bovinos de corte, correlação genética, herdabilidade.

\section{ABSTRACT}

There were evaluated at post weaning phase, 28.349 Aberdeen Angus breed animals, born from 1993 to 2003 on 141 farms, to estimate genetic parameters and to evaluate genetic and phenotypic trends for visual scores (EVs) conformation $(C)$, precocity $(P)$, musculature $(M)$ and size $(T)$. The covariance components were obtained by REML using an animal model. The heritabilities estimated were: 0.13, 0.11, 0.16 and 0.13 , to $C, P, M$ and $T$, respectively. The genetic correlations between the EVs range from 0.01 to 0.92 . The genetic and phenotypic trends estimated for $C, P, M$, and $T$ (points/year) were 0.0054 and $0.0189 ; 0.0035$ and -0.0013 ; 0.0057 and 0.0217; and 0.0026 and -0.0016 , respectively. The heritabilities estimated showed low responses by direct selection. The genetic correlations between the EVs were high between $C, P$ and $M(0.79$ to 0.92) and low between these and $T$ (0.01 to 0.30). The genetic trends showed that, selection is promoting low genetic progress; however the phenotypic trends that are negative for some characteristics, indicate that more attention for environmental conditions must be given.

Key words: beef cattle, genetic evaluation, genetic correlations, heritabilities.

\section{INTRODUÇÃO}

A produção de carne no Brasil é uma atividade econômica de grande importância, reconhecida pela qualidade e pelo volume produzido. A competitividade e a crescente exigência dos mercados consumidores têm feito com que a eficiência da cadeia produtiva melhore, aumentando a produtividade e a qualidade dos produtos (CORRÊA et al., 2006). Nesse contexto, a seleção genética, aliada a melhorias nas condições de criação, tem sido uma importante ferramenta disponibilizada aos criadores, sendo que a escolha dos indivíduos com base no seu mérito genético é, segundo GARCIA et al. (2003), a forma mais comum e, certamente, a mais eficiente de promover o melhoramento genético dos rebanhos.

'Programa de Pós-graduação em Zootecnia, Universidade Federal de Santa Maria (UFSM), 97105-900, Santa Maria, RS, Brasil. Email: samotweber@hotmail.com.*Autor para correspondência.

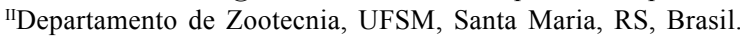

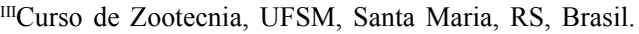


Para que melhorias genéticas sejam promovidas no rebanho nacional, além das características a serem melhoradas, é necessário que o ganho genético provável a ser promovido pelos reprodutores seja conhecido e, para isso, é indispensável o conhecimento da herdabilidade das características, bem como das correlações genéticas entre elas. Dentre as características de desempenho, as características da fase pós-desmama são importantes porque é nessa fase em que o desempenho do animal depende apenas do seu próprio genótipo e da ação do meio ambiente, tendo os efeitos maternos pouca ou nenhuma influência.

A utilização de escores, obtidos pela avaliação visual, é considerada uma boa forma para identificar animais de melhor conformação produtiva (KOURY FILHO, 2005) e, segundo FRIES (1996), essa pode ser uma das maneiras mais viáveis para quantificar as diferenças entre indivíduos, uma vez que, de acordo com DIBIASE et al. (2006), esse sistema, além de possibilitar a descrição física e a descrição produtiva do animal, permite estabelecer programas de seleção com ênfase em características que também precisam ser melhoradas em um rebanho. Segundo FORNI et al. (2007), resultados de pesquisa indicam que a seleção baseada em escores de avaliação visual podem promover mudanças genéticas efetivas em rebanhos de gado de corte, visto que as estimativas de herdabilidade obtidas para essas características apresentam magnitude média e suas correlações genéticas estimadas com os pesos em diferentes idades são favoráveis. No entanto, na literatura, são poucos os trabalhos sobre esse tema.

A eficiência dos programas de seleção depende da identificação e da utilização de material geneticamente superior para as características em questão, assim, é necessário que o programa seja periodicamente avaliado para verificar sua eficiência. Esse monitoramento pode ser feito por meio da avaliação da mudança genética ao longo do tempo, visando não só verificar o progresso genético alcançado, mas também realizar os ajustes necessários (EUCLIDES FILHO et al., 2000).

Este estudo teve por objetivos estimar parâmetros genéticos, tais como os coeficientes de herdabilidade e de correlação genética, para os escores de avaliação visual na fase pós-desmama, bem como avaliar as tendências genéticas e fenotípicas em uma população bovina da raça Aberdeen Angus.

\section{MATERIAL E MÉTODOS}

Os dados utilizados para a realização deste estudo foram fornecidos pela Associação Nacional de Criadores "Herd Book Collares" (ANC) e foram coletados em rebanhos da raça Aberdeen Angus controlados pelo Programa de Melhoramento de Bovinos de Carne (PROMEBO). As características avaliadas foram os escores, obtidos por avaliação visual, para conformação (C), que estima a quantidade de carne na carcaça do animal caso tenha sido abatido naquele momento pelo comprimento e pela profundidade do corpo, em conjunto com o desenvolvimento muscular e a harmonia geral do indivíduo; a precocidade $(\mathrm{P})$, que estima a capacidade do indivíduo de chegar a um acabamento mínimo de carcaça com peso vivo não elevado; a musculatura (M), que representa o grau de desenvolvimento muscular apresentado pelo animal; o tamanho (T), que compreende o comprimento e a altura do corpo do animal. As avaliações foram feitas em escala de um a cinco, em que o valor cinco é atribuído aos animais com o grau máximo de expressão, para determinada característica, dentro de um grupo de contemporâneos ao sobreano. A idade média na ocasião da avaliação foi 546 dias (mínimo 272 e máximo 814 dias), sendo que apenas cerca de $1 \%$ dos animais tinha idade inferior a um ano.

Foram formados grupos de contemporâneos $(\mathrm{GC}=712)$, reunindo os animais nascidos na mesma fazenda, no mesmo ano e na mesma estação, além de pertencerem ao mesmo sexo. A data juliana da desmama (DJD) foi criada em classes de 20 dias cada, formando um total de 20 classes.

Foram eliminados do arquivo original os registros incompletos, como a falta da identificação do pai ou da mãe, do registro do escore de avaliação visual, dos registros de produtos de vacas com idade menor que dois e maior que 16 anos; animais cujo tipo de reprodução não tivesse sido por inseminação artificial ou por monta dirigida, grupos de contemporâneos com menos de 10 animais e touros com menos de 10 filhos.

$\mathrm{O}$ arquivo de trabalho ficou constituído de registros tomados em 28.349 animais, nascidos entre os anos de 1993 e 2003 e criados em 141 fazendas, filhos de 753 touros e de 19.968 vacas.

As variáveis incluídas nos modelos foram definidas por meio de uma análise de variância, considerando um nível de significância de 5\%, utilizando o procedimento GLM (SAS, 2001), que considerou $\mathrm{C}, \mathrm{P}, \mathrm{M}$ e T em função dos efeitos fixos de GC e data juliana de desmama (DJD), além das co-

Ciência Rural, v.39, n.3, mai-jun, 2009. 
variáveis peso à desmama $(\mathrm{PD})$ e idade ao sobreano (IS), com efeitos linear e quadrático.

Os componentes de (co)variância utilizados para estimar a herdabilidade direta foram obtidos pelo método da Máxima Verossimilhança Restrita Livre de Derivada, com o programa MTDFREML, descrito por BOLDMAN et al. (1995). A matriz de parentesco foi constituída de 49.070 animais. Os modelos de análise utilizados são descritos, sob forma matricial, como: $y=$ $X \beta+Z a+e$ sendo $\mathrm{y}=$ vetor das observações de cada característica $\mathrm{C}, \mathrm{P}, \mathrm{M}$ e $\mathrm{T} ; X=$ matriz de incidência associada aos efeitos fixos; $\beta=$ vetor de solução para os efeitos fixos; $Z$ = matriz de incidência associada ao efeito genético aditivo direto de cada animal; $a=$ vetor de soluções para os efeitos genéticos aditivos diretos aleatórios; $e=$ vetor dos resíduos. O modelo utilizado foi o mesmo proposto por CARDOSO et al. (2004) para escores visuais na fase pós-desmama, não considerando o efeito materno, uma vez que este não apresenta efeito significativo. CARDOSO et al. (2001), avaliando escores visuais na fase pré-desmama em bovinos Angus, não incluíram no modelo de análise o efeito materno, visto que este, em análise prévia dos dados, apresentou valores de herdabilidade próximos de zero.

As tendências genéticas para as características analisadas foram obtidas pela regressão dos valores genéticos sobre o ano de nascimento dos animais; foram utilizadas as médias aritméticas dos valores genéticos de cada grupo de animais dentro de seu respectivo ano de nascimento, ponderadas pelo número de observações. Para tanto, foi utilizado o procedimento PROC REG do SAS (2001), com a equação: $Y_{i}=b_{o}+b_{1} X_{i}+e_{i}$ sendo $Y_{i}=$ Valor genético para as características avaliadas do $\mathrm{i}^{\text {ésimo }}$ ano de nascimento; $b_{o}=$ intercepto; $b_{1}=$ coeficiente angular da reta; $x_{i}=\mathrm{i}^{\text {śsiono }}$ ano de nascimento; $e_{i}=$ erro aleatório.

\section{RESULTADOS E DISCUSSÃO}

As variâncias genéticas aditivas estimadas (Tabela 1) representaram de 11,3 a 15,5\% da variância fenotípica apenas, evidenciando uma grande influência do ambiente sobre esses escores visuais, o que pode ser verificado pela magnitude da variância ambiental em relação à fenotípica $(88,7$ a $84,5 \%)$. Variâncias genéticas aditivas maiores foram relatadas por CARDOSO et al. (2004) para animais da mesma raça (respondendo por 18,6 a 25,28\% da variância fenotípica para os mesmos escores visuais).

As estimativas de herdabilidade para os escores de avaliação visual ao sobreano apresentaram valores baixos (Tabela 1), sendo o menor valor
Tabela 1 - Estimativas dos componentes de variância e herdabilidade para escores visuais de conformação (C), precocidade $(\mathrm{P})$, musculatura $(\mathrm{M})$ e tamanho (T) na fase de pós-desmama, obtidos por análises univariadas.

\begin{tabular}{lcccc}
\hline & $\sigma^{2}{ }_{a}$ & $\sigma^{2}$ & $\sigma^{2}$ & $h^{2}$ \\
\hline $\mathrm{C}$ & 0,08 & 0,57 & 0,66 & $0,13 \pm 0,01$ \\
$\mathrm{P}$ & 0,08 & 0,61 & 0,69 & $0,11 \pm 0,01$ \\
$\mathrm{M}$ & 0,10 & 0,56 & 0,66 & $0,16 \pm 0,02$ \\
$\mathrm{~T}$ & 0,08 & 0,53 & 0,61 & $0,13 \pm 0,02$ \\
\hline
\end{tabular}

$\sigma_{\mathrm{a}}^{2}=$ variância genética aditiva, $\sigma_{\mathrm{e}}^{2}=$ variância ambiental, $\sigma_{\mathrm{p}}^{2}=$ variância fenotípica, $\mathrm{h}^{2}=$ herdabilidade \pm erro padrão da estimativa; Variâncias em pontos ${ }^{2}$ para C, P, M e T.

observado para $\mathrm{P}(0,11)$ e o maior valor estimado para $\mathrm{M}(0,16)$, enquanto $\mathrm{C}$ e $\mathrm{T}$ apresentaram valores iguais $(0,13)$, similares aos valores obtidos por COSTA $(2005)$ para a raça Brangus $(0,12,0,14 \mathrm{e} 0,13$ respectivamente para C, $\mathrm{Pe} \mathrm{M}$ ). PONS et al. (1989) relataram valor maior $(0,52)$ para $C$, para a raça Hereford, da mesma forma que CARDOSO et al. (2004) encontraram valores de 0,19, $0,25,0,26$ e 0,24 , respectivamente, para C, P, M e T, para a raça Angus. ROSO \& FRIES (1995) encontraram valores menores aos do presente estudo para as características M e T $(0,04$ e 0,03), respectivamente, no entanto, para $C$, estimaram valor maior $(0,39)$ para animais da raça Polled Hereford.

Embora as estimativas de herdabilidade obtidas indiquem a possibilidade de ganho genético, este deve ser esperado em pequena proporção. A grande variabilidade das estimativas encontradas neste estudo e na literatura, no que se refere às características com base em avaliação visual, pode, em parte, ser devida a inconsistências nos sistemas de avaliação ou diferenças genéticas entre os indivíduos das populações. Segundo CARDOSO et al. (2004), é possível aumentar a precisão da seleção para os escores visuais ao sobreano por meio do melhor controle das condições ambientais, como a melhor definição dos grupos de contemporâneos, as melhores condições alimentares e sanitárias e, ainda, a maior padronização nas avaliações subjetivas dos animais.

As correlações genéticas (Tabela 2 ) entre $\mathrm{C}$ e $\mathrm{P}$ e entre $\mathrm{C}$ e $\mathrm{M}$ foram altas $(0,83$ e 0,92 , respectivamente), concordando com CARDOSO et al. (2004), os quais afirmaram que, pelo fato de que na avaliação da conformação é considerada a musculatura, o tamanho e o acabamento do animal, há certa redundância em avaliar $\mathrm{C}$, quando já foram observados $\mathrm{P}, \mathrm{M} \mathrm{e} \mathrm{T}$.

O valor obtido para a correlação genética entre $\mathrm{C}$ e $\mathrm{T}$ foi médio $(0,30)$. As correlações genéticas 
Tabela 2 - Estimativas dos componentes de covariância (acima da diagonal) e correlações genéticas (abaixo da diagonal) para os escores visuais de conformação (C), precocidade $(\mathrm{P})$, musculatura $(\mathrm{M})$ e tamanho $(\mathrm{T})$ na fase de pós-desmama, obtidas por análises bivariadas.

\begin{tabular}{lcccc}
\hline & $\mathrm{C}$ & $\mathrm{P}$ & $\mathrm{M}$ & $\mathrm{T}$ \\
\hline $\mathrm{C}$ & - & 0,07 & 0,09 & 0,02 \\
$\mathrm{P}$ & 0,83 & - & 0,07 & 0,00 \\
$\mathrm{M}$ & 0,92 & 0,79 & - & 0,01 \\
$\mathrm{~T}$ & 0,30 & 0,01 & 0,09 & - \\
\hline
\end{tabular}

mais baixas ocorreram entre $\mathrm{P}$ e $\mathrm{T}(0,01)$ e entre $\mathrm{Me} \mathrm{T}$ $(0,09)$, indicando que essas características são geneticamente independentes, ou seja, não são controladas pelos mesmos grupos de genes, no entanto, não são antagônicas, como se poderia esperar, pressupondo que os maiores animais são os de acabamento mais tardio. O mesmo comportamento das correlações genéticas obtidas entre os escores visuais foi relatado por CARDOSO et al. (2004), porém, com valores de maior magnitude do que os encontrados neste estudo.

As tendências genéticas obtidas para os escores visuais (Figura 1$)$ foram todas positivas $(0,0054$, $0,0035,0,0057$ e 0,0026 pontos/ano) e significativas $(\mathrm{P}<0,0001)$, representando 0,16, 0,10, 0,17 e 0,08\% das médias fenotípicas de $\mathrm{C}, \mathrm{P}, \mathrm{M}$ e $\mathrm{T}$, respectivamente. $\mathrm{O}$ menor ganho genético anual foi observado para $\mathrm{T}$ $(0,0026$ pontos/ano $)$, talvez pelo fato deste ter correlação genética menor com os outros escores. Os escores $\mathrm{C}$ e $\mathrm{M}$ apresentaram os maiores ganhos genéticos, cabendo ressaltar que a correlação genética entre eles foi a mais alta $(0,92)$. Não foram encontradas, na literatura, estimativas de tendências genéticas para $\mathrm{C}, \mathrm{P}, \mathrm{M}$ e $\mathrm{T}$ em bovinos de raças taurinas, na pósdesmama, que servissem como base para comparações. No entanto, para a raça Nelore, VAN MELIS et al. (2003) relataram valores de tendência genética maiores que os encontrados neste estudo para os escores de $\mathrm{C}$ $(0,030), \mathrm{P}(0,031)$ e $\mathrm{M}(0,030)$.

Apesar de os ganhos genéticos obtidos apresentarem valores baixos, o progresso genético deve ser considerado, pois as mudanças genéticas são estáveis, cumulativas e permanentes ao longo dos anos, entretanto, apesar de serem estáveis, tais mudanças genéticas podem vir a não se expressar devido a condições ambientais distintas ao longo dos anos avaliados. Dessa forma, espera-se que a utilização de índices de seleção para tais características proporcione ganhos genéticos de maior magnitude, visto que esses índices consideram as características em conjunto e com valores relativos a sua importância econômica.
As tendências fenotípicas apresentadas para C e M (Figura 2) apresentaram a mesma direção da tendência genética, entretanto, os ganhos fenotípicos foram maiores em relação aos ganhos genéticos $(0,0189$ e 0,0217 pontos/ano para $\mathrm{C}$ e $\mathrm{M}$, respectivamente). Contudo, em virtude dos baixos ganhos genéticos apresentados para essas características, pode-se deduzir que os aumentos das médias fenotípicas ocorreram principalmente pela melhoria nos fatores de meio que atuam sobre os animais.

Para $\mathrm{P} \mathrm{e} \mathrm{T,} \mathrm{as} \mathrm{tendências} \mathrm{fenotípicas} \mathrm{(Figura}$ 2) apresentaram comportamento antagônico à
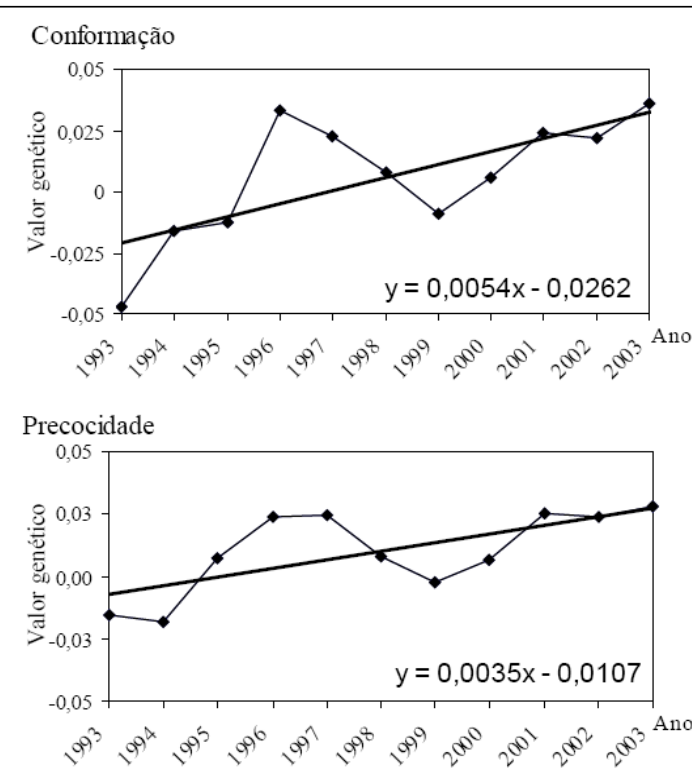

Musculatura

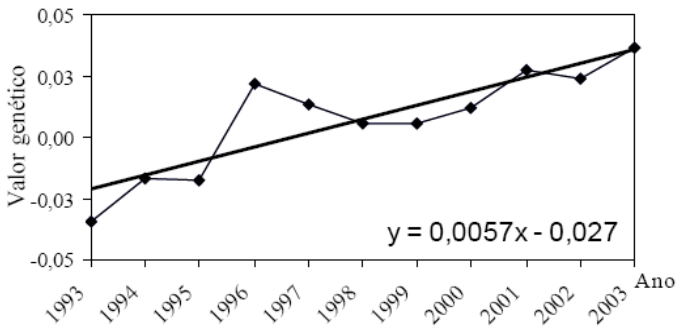

Tamanho

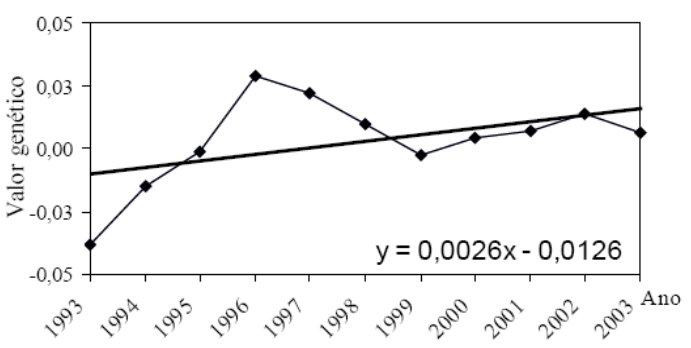

Figura 1 - Tendências genéticas, em pontos, para os escores visuais de conformação $(\mathrm{C})$, precocidade $(\mathrm{P})$, musculatura $(\mathrm{M})$ e tamanho $(\mathrm{T})$ para uma população da raça Aberdeen Angus.

Ciência Rural, v.39, n.3, mai-jun, 2009. 


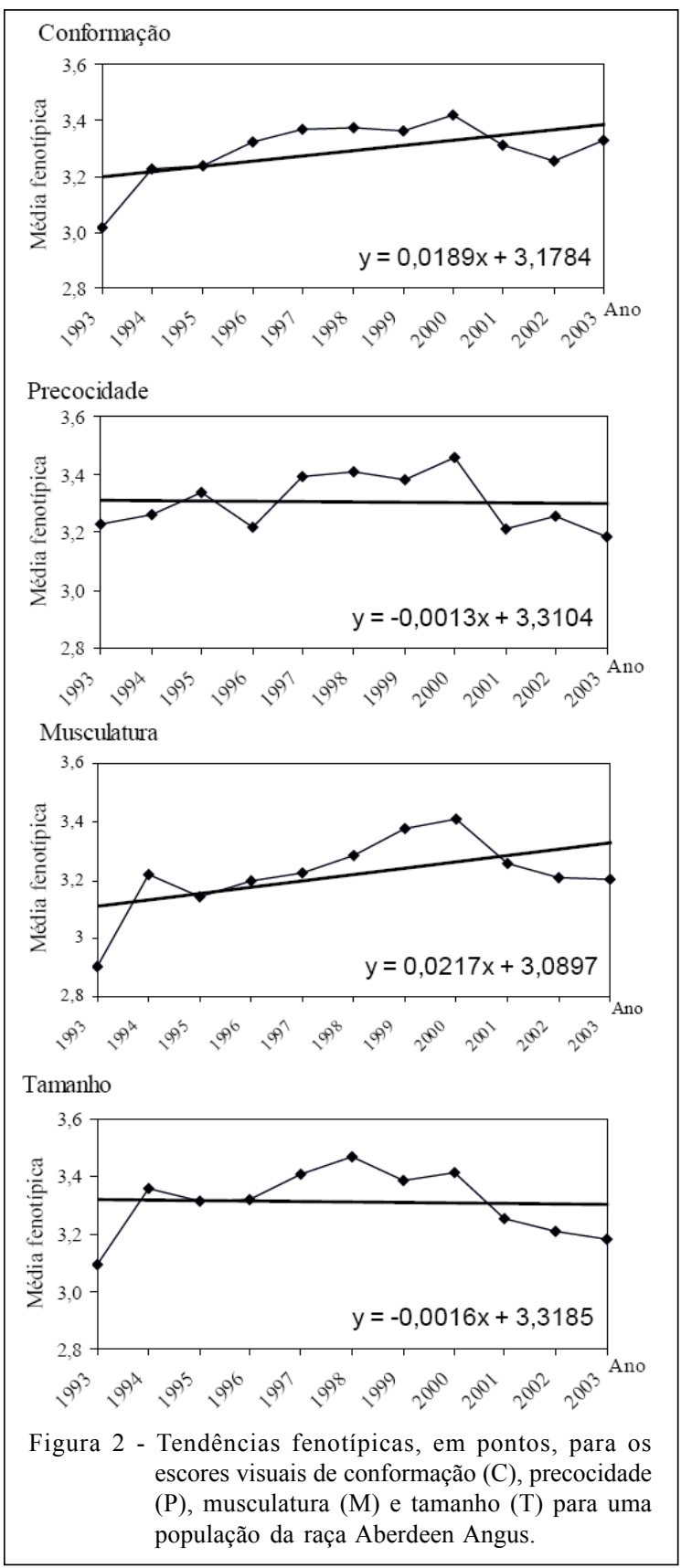

tendência genética, sendo decrescentes e iguais a $-0,0013$ e $-0,0016$ pontos/ano, respectivamente (significativos $\mathrm{P}<0,0001$ ), indicando que os animais não conseguiram expressar o seu potencial genético, visto que o ganho genético foi crescente, e a média fenotípica foi decrescente ao longo dos anos. Embora as quatro características tenham sido avaliadas concomitantemente, elas são determinadas, parcialmente, por grupos de genes diferentes, os quais respondem com intensidades diferentes ao efeito de um mesmo ambiente. Além disso, a resposta fenotípica pode também ter sido afetada por possíveis inconsistências no processo de avaliação visual dos animais.

\section{CONCLUSÕES}

A seleção por escores visuais para conformação, musculatura, precocidade e tamanho promove ganho genético de pequena magnitude; portanto, além do desempenho individual, é importante que a seleção seja feita com informações de parentesco e/ou teste de progênie. As altas correlações genéticas entre alguns escores e baixas entre outros indicam que maior resposta à seleção pode ser obtida por meio da utilização de Índices de Seleção, combinando essas características. As tendências genéticas indicam a existência de progresso genético para as características avaliadas, no entanto, melhorias ambientais devem ser realizadas conjuntamente com o melhoramento genético a fim de permitir a expressão do verdadeiro potencial genético dos animais.

\section{REFERÊNCIAS}

BOLDMAN, K.G.et al. A manual for use of MTDFREML. A set of program to obtain estimates of variances and covariances (DRAFT). Lincoln: Departament of Agriculture/ Agricultural Research Servica, 1995. 120p.

CARDOSO, F.F. et al. Componentes de (Co)variância e parâmetros genéticos para caracteres produtivos à desmama de bezerros Angus Criados no estado da Rio Grande do Sul. Revista Brasileira de Zootecnia, v.30, n.1, p.41-48, 2001.

CARDOSO, F.F. et al. Componentes de (co)variância e parâmetros genéticos de caracteres pós-desmama em bovinos da raça Angus. Revista Brasileira de Zootecnia, v.33, n.2, p.313-319, 2004.

COSTA, G.Z. Estudo de escores visuais e de ganhos médios diários de peso de animais formadores da raça Brangus. 2005. 70f. Dissertação (Mestrado em Zootecnia - Genética e Melhoramento Animal) - Faculdade de Ciências Agrárias e Veterinárias, Universidade Estadual Paulista.

CORRÊA, M.B.B. et al. Influência ambiental sobre características de desempenho pré-desmama de bovinos Devon no Rio Grande do Sul. Revista Brasileira de Zootecnia, v.35, n.3, p.1005-1011, 2006 (supl.).

DIBIASI, N.F. Estudo do crescimento, avaliação visual, medidas por ultrasonografia e precocidade sexual em touros jovens pertencentes a vinte e uma raças com aptidão para corte. 2006. 75f. Dissertação (Mestrado em Zootecnia - Genética e Melhoramento Animal). Faculdade de Ciências Agrárias e Veterinárias, Universidade Estadual Paulista, Jaboticabal.

EUCLIDES FILHO, K. et al. Tendências genéticas na raça Gir. Pesquisa Agropecuária Brasileira, v.35, n.4, p.787-791, 2000 .

Ciência Rural, v.39, n.3, mai-jun, 2009. 
FORNI, S. et al. Tendências genéticas para escores visuais de conformação, precocidade e musculatura à desmama de bovinos Nelore. Revista Brasileira de Zootecnia, Viçosa, v.36, n.3, p.572-577, 2007

FRIES, L.A. Uso de escores visuais em programas de seleção para a produtividade em gado de corte. In: SEMINÁRIO NACIONAL - REVISÃO DE CRITÉRIO DE JULGAMENTO E SELEÇÃO EM GADO DE CORTE. 1996, Uberaba. Anais... Uberaba: ABCZ, 1996. p.1-6.

GARCIA, F.Q. et al. Tendência dos efeitos genéticos diretos e maternos do peso a desmama de bovinos da raça Nelore Mocha na região pecuária Campo Grande e Dourados - Mato Grosso do Sul. Archives of Veterinary Science, v.8, n.1, p.93-97, 2003.

KOURY FILHO, W. Escores visuais e suas relações com características de crescimento em bovinos de corte. 2005 80f. Tese (Doutorado em Zootecnia - Produção Animal) Faculdade de Ciências Agrárias e Veterinárias, Universidade Estadual Paulista.

PONS, S.B. et al. Efeito de fatores genéticos e de ambiente sobre o crescimento e o escore de conformação em bovinos Hereford no Rio Grande do Sul. I - Peso e escore de conformação à desmama. Revista Brasileira de Zootecnia, v.18, n.5, p.402-409, 1989.

ROSO, V.M.; FRIES, L.A. Componentes principais em bovinos da raça Polled Hereford à desmama e sobreano. Revista Brasileira de Zootecnia, v.24, n.5, p.728-735, 1995.

SAS. SAS user's guide: statistical. ACary, NC, 2001. 438p.

VAN MELIS, M.H. et al. Estimação de parâmetros genéticos em bovinos de corte utilizando os métodos de máxima verossimilhança restrita e $\mathfrak{R}$. Revista Brasileira de Zootecnia, v.32, n.6, p.1624-1632, 2003. (Supl. 1). 